\title{
Intermedin/adrenomedullin2: an autocrine/paracrine factor in vascular homeostasis and disease
}

\author{
NI XianQiang ${ }^{1,2,3}$, ZHANG JinSheng ${ }^{1,2,3}$, TANG ChaoShu ${ }^{1,2} \&$ QI YongFen ${ }^{1,2,3 *}$ \\ ${ }^{1}$ Laboratory of Cardiovascular Bioactive Molecule, School of Basic Medical Sciences, Peking University, Beijing 100191, China; \\ ${ }^{2}$ Key Laboratory of Molecular Cardiovascular Science, Ministry of Education, Peking University Health Science Center, Beijing 100191, \\ China; \\ ${ }^{3}$ Department of Microbiology and Parasitology, School of Basic Medical Science, Peking University, Beijing 100191, China
}

Received May 14, 2014; accepted June 20, 2014

\begin{abstract}
Intermedin (IMD) or adrenomedullin 2 is a novel peptide related to the calcitonin gene-related peptide (CGRP) family. Via calcitonin receptor-like receptor/receptor activity modifying proteins, the common receptor complexes of CGRP, IMD exerts a wide range of biological effects, especially regulation of cardiovascular homeostasis. Proteolytic processing of a larger IMD precursor yields a series of biologically active C-terminal fragments, IMD1-53, IMD1-47 and IMD8-47. IMD and its receptors are present in the cardiovascular system, and IMD is present at low levels in plasma. In the cardiovascular system, IMD has multiple functions such as regulation of blood pressure and cardiac function, pro-angiogenesis, endothelial barrier function protection, anti-oxidative stress, and anti-endoplasmic reticulum stress. IMD participates widely in the pathogenesis of atherosclerosis, hypertension, pulmonary arterial hypertension and vascular calcification. It is a vascular regulatory factor of homeostasis and a vital endogenous protective factor against vascular diseases.
\end{abstract}

intermedin, vessel, homeostasis, vascular diseases

Citation: Ni XQ, Zhang JS, Tang CS, Qi YF. Intermedin/adrenomedullin2: an autocrine/paracrine factor in vascular homeostasis and disease. Sci China Life Sci, 2014, 57: 781-789, doi: 10.1007/s11427-014-4701-7

Intermedin (IMD) or adrenomedullin 2 (ADM2) is a novel biologically active polypeptide independently discovered in 2004 [1,2]. Subsequently, the two peptides were found to have the same amino acid sequence and similar biological function, so they were considered the same substance. IMD is a novel member of the calcitonin/calcitonin gene-related peptide (CGRP) family, which includes calcitonin, CGRP, amylin, and ADM [1]. Their encoding genes are highly homologous and they play an important role in regulating the body homoeostasis and cardiovascular disease. IMD usually has similar biological effects as other members of the CGRP family, such as anti-oxidative stress effects, positive inotropic action, natriuretic and diuretic effects, regu-

*Corresponding author (email: yongfenqi@ 163.com) lating hormone secretion, neurotransmission or neuromodulation, and anti-apoptosis effects [3-10]. However, IMD actions differ from those of ADM. For instance, with a rat renal-impairment model prepared by $5 / 6$ nephrectomy in Wistar Kyoto (WKY) rats, Hirose et al. [11] found IMD expression upregulated and that of ADM downregulated in the kidneys, so under certain pathological conditions, the pathophysiologic functions of IMD and ADM differ. The different biological functions for IMD and other members of the CGRP family may be related to the wider tissue distribution of IMD in the body, non-selectivity of IMD to CGRP family receptors and a possible specific IMD antibody.

In recent years, growing evidence has indicated that IMD is a vital bioactive peptide maintaining vascular homeosta- 
sis and has extensive functions in the cardiovascular system, such as regulation of blood pressure and cardiac function, accelerating angiogenesis, and anti-apoptosis and powerful cardiovascular protective functions [9,12-14]. IMD is widely involved in the pathogenesis of vascular disease such as hypertension, vascular calcification and atherosclerosis [15-17]. In this review, we summarize the effects of IMD on the cardiovascular system, the relationship between IMD and vascular diseases and the possible molecular mechanism maintaining vascular homeostasis.

\section{Biochemical characteristics of IMD}

\subsection{Molecular structure of IMD}

Human IMD gene is located on the distal arm of chromosome 22 and encodes a prepro-peptide of 148 amino acids, called prepro-IMD. Basic proteolytic cleavage sites of prepro-IMD are found at Arg100-Thr101 and Arg107-Val108; proteolytic cleavage at these sites followed by cleavage of Tyr147-Gly148 and amidation of the C-terminal amino acid yields a long 47 amino acid form of the peptide IMD1-47 (prepro-IMD101-147) and a short 40 amino acid peptide IMD8-47 (prepro-IMD108-147) [1]. Subsequently, our laboratory identified another proteolytic cleavage site in rat prepro-IMD between Arg92-His93 and obtained an additional larger peptide IMD1-53 (prepro-IMD93-145), which may be the main active fragment of IMD [18] (Figure 1).

IMD is highly conserved between different animal species. The mouse and rat IMD1-47 molecules differ in one amino acid; the human IMD1-47 amino acid sequence is $87 \%$ identical to that of rat and $>60 \%$ identical to that of osteichthyes. The structure of IMD is more similar to ADM and CGRP than amylin (AMY) and calcitonin (CT), so IMD, ADM and CGRP are probably a sub-branch in the evolution of the CGRP superfamily [1]. IMD has a similar protein structure with members of the CGRP family, namely, an
$\mathrm{N}$-terminal intramolecular loop of six amino acids flanked by a disulphide bond, followed by an amidation structure of a C-terminal amide. These two special structures are important for the IMD biological effects.

\subsection{Distribution and production of IMD}

The main active fragments of IMD in plasma are IMD1-53, IMD1-47 and IMD8-47. The sensitivity of the body greatly affects plasma IMD levels [19]. The primary source of plasma IMD is not exactly known; pituitary secretion may be the main source [1].

Transcriptional expression and immunohistochemical investigation in rodents and humans revealed particularly high levels of IMD in the kidney, gastrointestinal tract (especially the muscularis mucosa of the stomach) [1] and jejunum, brain (especially hypothalamus), skin, and submaxillary gland. IMD levels are relatively high in the pancreas, lung, spleen, thymus and ovaries but low in the adrenal gland and testis [1,2]. In the mature mouse heart, prepro-IMD is expressed at low levels, only in endothelial cells of the coronary artery and vein, and scarcely in myocardial cells [2]. The expression of IMD in healthy myocardial cells is low and is markedly upregulated under stress [20].

The relative paucity of IMD in the vicinity of the vasculature indicates that IMD is primarily an endocrine peptide. The prominent distribution of IMD in the renal tubule suggests a physiological role in local regulation of the water-electrolyte balance and circulatory blood volume. Robust expression in the diseased myocardium as compared with sparse levels in the healthy myocardium supports an important role in pathogenesis of the heart.

\subsection{IMD receptors and signaling transduction pathway}

IMD belongs to the CGRP superfamily of peptides. The common receptors of the CGRP family are calcitonin re-

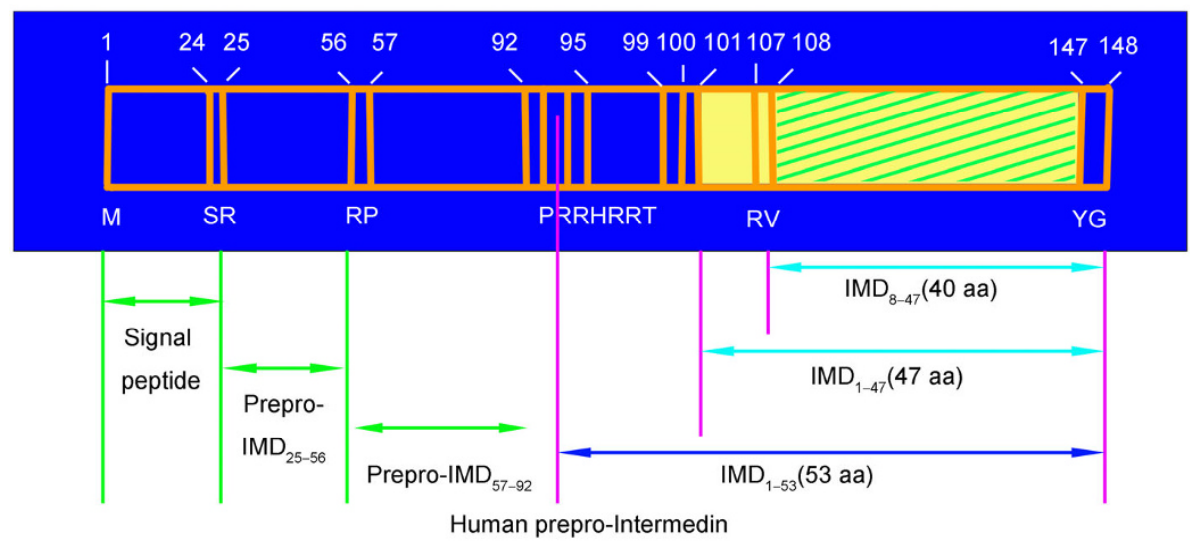

Figure 1 (color online) Schematic diagram of human prepro-intermedin, which generates different fragments derived from the proteolytic cleavage of 148 amino-acid prepropeptide precursor at the putative cleavage sites indicated. 
ceptor-like receptor/receptor activity modifying protein (CRLR/RAMP) complexes [1]. RAMPs act as molecular chaperones for trafficking CRLR from the endoplasmic reticulum and Golgi apparatus to the cell surface and have pivotal influence over the receptors' pharmacological characteristics. Given that RAMPs have three subtypes, there are three kinds of receptors. According to the affinity to CGRP and ADM, CRLR with RAMP1 offers the CGRP1 receptor; with RAMP2 or RAMP3, it offers the ADM1 or ADM2 receptor. IMD binds non-selectively to all three CRLR/RAMP complexes [1]. As compared to CGRP, IMD is less potent at CGRP1 receptors but more potent at ADM1 and ADM2 receptors; as compared with ADM, IMD is more potent at CGRP1 receptors but less potent at ADM1 and ADM2 receptors. CRLR belongs to the G-proteincoupled receptor (GPCR) superfamily and has a complex downstream signaling pathway. Stimulating adenylate cyclase $g$ protein Gs-mediated activation of adenylate cyclase-cyclic adenosine monophosphate-protein kinase A (AC-cAMP-PKA) represents the major signalling pathway coupled with these receptors [16,21-24]. In addition, Gq/11-mediated coupling to phospholipase C-b, Gi and/or Go-mediated regulation of potassium channels, and activation of phosphatidylinositol 3-kinase-guanylate cyclasecyclic guanosine monophosphate (PI3K-GC-cGMP) have been reported as downstream signaling pathways of IMD receptors $[10,13,15,25-27]$.

However, no specific receptor for IMD has been found. A large amount of evidence showed that IMD and ADM share many similar biological functions and IMD is stronger than ADM, but bonding to CRLR/RAMPs is not more potent than for ADM. Antagonists of CGRP and ADM can block or abolish the biological effects of IMD in vivo. Like other members of the CGRP family, IMD exerts its biological effect mainly through the common receptors CRLR/ RAMPs. CRLR belongs to the superfamily of seven-transmembrane GPCRs [20]. It undergoes conformational changes, which results in coupling to Gs, activation of $\mathrm{AC}$ and accumulation of intracellular cAMP when activated by IMD. cAMP accumulation can activate PKA. ACcAMP-PKA forms the most important intracellular signaling pathway of IMD. IMD is well known to increase the content of intracellular cAMP and mediate a variety of biological effects such as myocardial contraction, vasodilatation, inhibition of vascular calcification, regulating cell proliferation, hypertrophy, migration, and apoptosis [21,22,28].

The L-arginine-nitric oxide (NO)-cGMP signal pathway also plays an important role in the biological function of IMD. IMD treatment increased the activity of NO synthase (NOS) and content of NO in tissues and augmented the uptake of L-arginine by cells [29]. NO activates GC and elevates intracellular cGMP levels for multiple effects such as vasodilation, anti-oxidative stress, and inhibition of cell apoptosis [8,30,31]. IMD1-53 significantly increased NO production and endothelial NOS (eNOS) activity in rat aortas and was more potent than equivalent ADM [32]. However, IMD and ADM had no effect on inducible NOS expression and activity. Otherwise, IMD1-53 dosedependently increased $\left[{ }^{3} \mathrm{H}\right] \mathrm{L}-\mathrm{Arg}$ transport, and its effect was more potent than an equivalent concentration of ADM. Semiquantitative RT-PCR revealed that IMD1-53 significantly increased the mRNA expression of cationic amino acid transport 1 (CAT-1) and CAT-2B. Thus, IMD1-53 may regulate vessel function homeostasis by upregulating the L-arginine-NOS-NO pathway. The pulmonary vasorelaxant response (PVR) to recombinant IMD in pulmonary arterial (PA) rings were found completely inhibited by endothelium removal and NO production inhibitors [27]. IMD-stimulated endothelial CRLR is coupled to release of NO, activation of GCs, and promotion of hyperpolarization through large-conductance calcium-activated $\mathrm{K}(+)$ channels in the rat main pulmonary artery. IMD had a negative inotropic effect on the isolated myocardium because of NO-cGMP pathway activation with concomitant thin myofilament desensitization by increased cTnI phosphorylation [25], which provides a coherent explanation for the previously reported contradictory results (positive or negative inotropic effect). In conscious rat models, the regional haemodynamic profile of IMD resembled that of ADM [30], and some of the vasodilator effects of IMD were mediated by ADM receptors and NO but not by K(ATP) channels. Using anaesthetic rat models, Abdelrahman et al. found that IMD dose-dependently decreased mean arterial pressure and increased heart rate; the depressive effect of IMD was not mediated via the L-arginine-NO pathway, production of prostanoids or opening of tetraethylammonium-sensitive $\mathrm{K}^{+}$ channels but was opposed by activity of the sympathetic nervous system [33]. IMD treatment attenuating hypoxic pulmonary vascular remodeling, activating the L-arginineNO pathway and inhibiting the endoplasmic reticulum stress-specific apoptosis pathway could be the mechanisms mediating the anti-proliferative and anti-apoptotic effects of IMD [29] (Figure 2).

\section{IMD and vascular diseases}

A large amount of data showed that the changed expression of IMD and its receptor is involved in the initiation and development process of various vascular diseases. IMD has a strong inhibitory effect on the development of these diseases and may be a new target for prevention and treatment of such disease.

\subsection{IMD and atherosclerosis}

IMD has protective effect on the endothelial barrier function, so IMD could have a powerful anti-inflammatory effect. 
IMD stabilized endothelial barriers in human umbilical vein endothelial cell (HUVEC) monolayers via CRLR/RAMP2 receptors [23]. These effects were mediated via cAMPmediated inactivation of contractility and strengthening of cell-cell adhesion. IMD increased the permeability of rat coronary microvascular endothelial cells (RCECs) and reduced that of HUVECs and rat aortic endothelial cells via CRLRs and cAMP [34]. IMD caused derangement of the actin cytoskeleton along with loss of vascular endothelial cadherin (VE-cadherin) in RCECs but caused rearrangement of the actin cytoskeleton and VE-cadherin at cell-cell junctions in HUVECs. IMD inactivated the RhoA-Rhokinase (Rock) pathway in both cell types. However, it inactivated Rac1 in RCECs but not HUVECs. Inhibition and rescue experiments demonstrated that both RhoA and Rac1 are required for RCEC barrier stability, whereas in HUVECs, the inhibition of RhoA/Rock signalling did not interfere with basal permeability. Therefore, the opposite effects of IMD on permeability of RCECs and HUVECs are due to differential regulation of actin cytoskeleton dynamics via RhoA and Rac1. Moreover, Rac1 activity is regulated by the RhoA/Rock pathway in RCECs but not HUVECs. Human aortic endothelial cells were found to express IMD mRNA and secreted IMD peptide [8]. IMD mRNA expression depended on metabolic conditions and was selectively regulated in a contrary fashion to ADM mRNA. IMD mRNA expression in endothelial cells is markedly sensitive to oxidative stress, and IMD peptide itself has anti-apoptotic activity in human endothelial cells. These data suggest that the role of IMD differs from that of ADM and that IMD has a protective function in human endothelial cells.

IMD has an inhibitory effect on the development of atherosclerosis. Pretreatment with IMD for six weeks prevented the progression of atherosclerotic lesions and the increase in wall thickness in the aorta [35]. Oil-red O staining of the entire aorta and the atherosclerotic aortic root section showed 2-fold decrease of atherogenic plaque. Serum lipid profiles such as total cholesterol and low-density lipoprotein (LDL) cholesterol levels were decreased and high-density lipoprotein cholesterol level was increased. Thus, exoge-

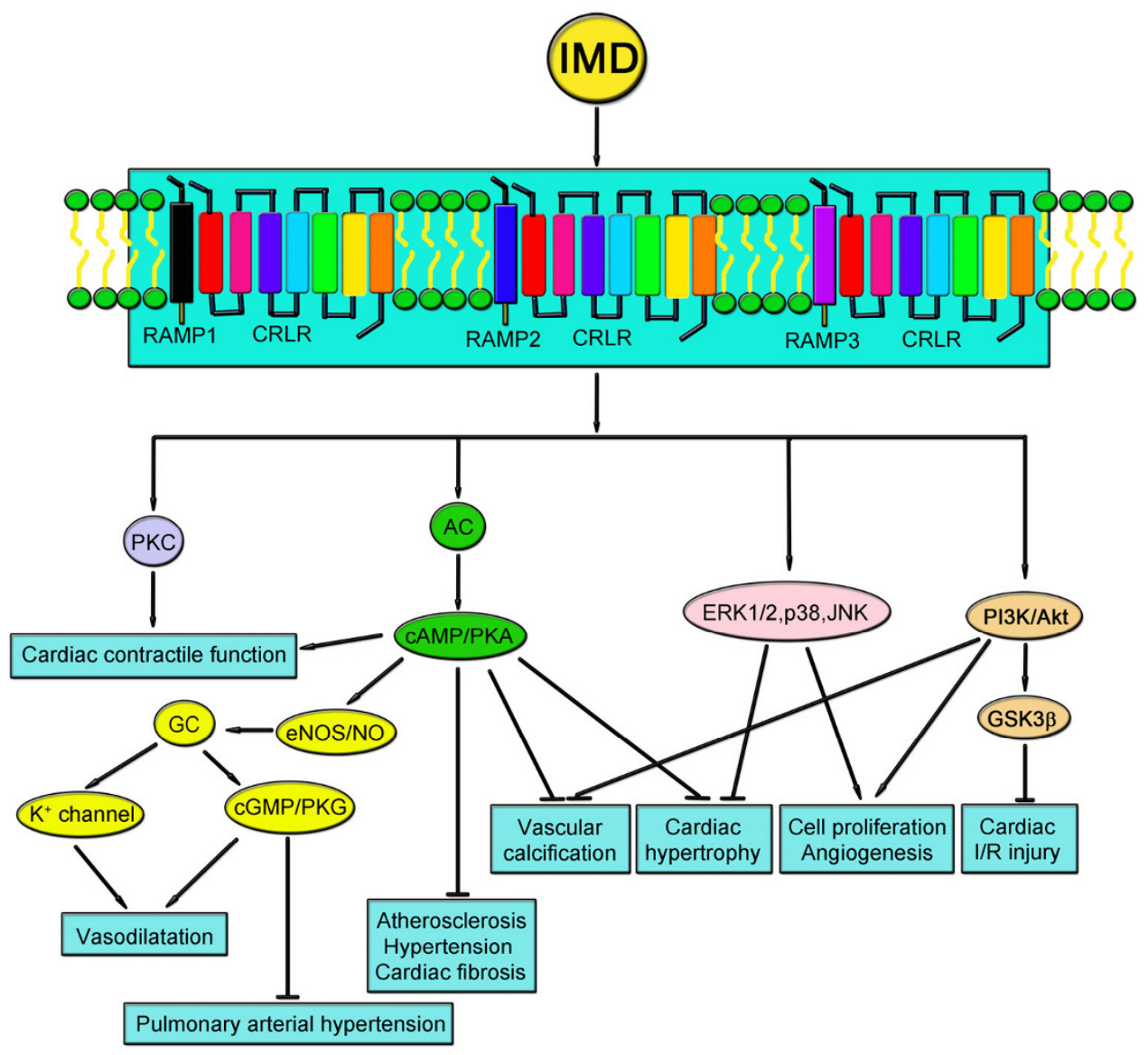

Figure 2 (color online) Schematic diagram outlining the signalling pathways by which IMD might evoke cardiovascular effects and be involved in protective effects on cardiovascular diseases. CRLR, calcitonin receptor-like receptor; RAMPs, receptor activity-modifying proteins; AC, adenylate cyclase; cAMP, cyclic adenosine monophosphate; PKA, protein kinase A; PKC, protein kinase C; GC, guanylate cyclase; cGMP, cyclic guanosine monophosphate; PKG, protein kinase G; eNOS, endothelial nitric oxide synthase; ERK, extracellular signal-regulated kinase; PI3K, phosphoinositide 3-kinase; Akt/PKB, protein kinase B; GSK-3 $\beta$, glycogen synthase kinase-3 beta. 
nous administration of IMD could prevent the progression of atherosclerotic plaque. The possible underlying mechanisms may relate to the improvement of lipid profiles. Dai et al. [36] revealed a new mechanism of the anti-atherogenic role of IMD and a novel pattern for regulation of CD36 expression in macrophages. In apolipoprotein E-deficient mice, six-week IMD infusion reduced oxidized LDL uptake, intracellular cholesterol content, and foam-cell formation in peritoneal macrophages as well as the protein and mRNA levels of CD36. These in vivo results agreed with in vitro observations in primary peritoneal macrophages. Reduced CD36 protein and mRNA levels were due to IMDaccelerated decay of CD36 mRNA. Tristetraprolin (TTP), which binds to AU-rich elements in the $3^{\prime}$ untranslated regions of mRNA and promotes its degradation, mediates CD36 mRNA destabilization. TTP knockdown by short-hairpin RNA increased and TTP overexpression reduced CD36 expression, and TTP knockdown rescued IMD-reduced CD36 expression. IMD attenuated macrophage foam-cell formation via TTP-mediated degradation of CD36 mRNA. In an in vitro foam-cell formation model (induced by acetylated LDL, Ac-LDL) with mouse (C57BL/6J) macrophages, IMD reduced Ac-LDL uptake and binding, decreased intracellular cholesterol content, and suppressed both mRNA and protein levels of scavenger receptor A (SR-A) [16]. Simultaneously, IMD increased phosphatase and tensin homolog (PTEN) protein levels by increasing PTEN phosphorylation and inhibiting ubiquitin-mediated PTEN degradation. These effects were blocked by the IMD receptor antagonist or cAMP-PKA inhibitors. PTEN overexpression mimicked the inhibitory effects of IMD on SR-A expression and Ac-LDL uptake. However, knockdown of PTEN by short-hairpin RNA completely blocked all inhibitory effects of IMD. Furthermore, in apolipoprotein E-deficient mice, six-week IMD infusion reduced Ac-LDL uptake and SR-A mRNA and protein levels and increased PTEN protein level in peritoneal macrophages. PTEN level was increased and SR-A expression decreased in parallel in macrophages in atherosclerotic lesions. Thus, IMD inhibited atherosclerosis in $a p o E^{-1-}$ mice. Increased stability of PTEN by IMD led to SR-A inhibition in macrophages, which ameliorated foam-cell formation and atherosclerosis in $a p o E^{-/}$mice.

\subsection{IMD and hypertension}

IMD also plays an important role in regulating vascular biology. Intraperitoneal injection of IMD1-47 had a hypotensive effect on rat blood pressure. The hypotensive response was markedly attenuated by treatment with CGRP8-37 in normotensive and spontaneously hypertensive rats (SHRs) [1]. Intravenous injection of IMD1-47 decreased arterial pressure more potently than did ADM in mice [2]. IMD (0.3-30 nmol kg-1) dose-dependently decreased mean arterial pressure and increased heart rate in anesthetized rats [33]. The depressive effect of IMD (3 nmol $\mathrm{kg}^{-1}$, ED70 dose) was not affected by pretreatment with an inhibitor of NOS, a cyclooxygenase inhibitor, or the nonspecific $\mathrm{K}^{+}$-channel blocker tetraethylammonium. Pretreatment with mecamylamine (ganglionic blocker) augmented the depressive response and abolished the tachycardic effect of IMD. Therefore, the depressive effect of IMD is not mediated via the L-arginine-NO pathway, the production of prostanoids or opening of tetraethylammonium-sensitive $\mathrm{K}^{+}$channels but is opposed by activity of the sympathetic nervous system. IMD, as a cardiovascular active peptide, was found involved in reducing blood pressure and ameliorating cardiac function during hypertension [24]. The mechanism underlying this effect may involve its binding of a receptor complex formed by CRLR and RAMPs and consequent regulation of cAMP levels. Exonic insertion (I)/deletion (D) polymorphism was found to influence the generation of IMD-53, a DD carrier, as compared with II and ID carriers, for a significantly higher 24-h artery blood pressure and lower estimated glomerular filtration rate [37]. The prevalence of chronic kidney disease as well as presence of lacunar infarction and white matter hyperintensity was greater for DD than II carriers (odds ratio 2.4, $P=0.01$ and $2.7, P=0.003$, respectively). The IMD I/D polymorphism was associated with renal dysfunction, blood pressure regulation and asymptomatic cerebrovascular diseases in the Japanese general population.

Site-specific microinjection of IMD bilaterally into the nucleus tractus solitarii (NTS) significantly increased renal sympathetic nerve activity (RSNA) and mean arterial pressure (MAP) [22]. IMD-evoked increases in renal sympathetic nerve activity and mean arterial pressure were abolished by pretreatment with the receptor antagonist ADM22-52, an AC inhibitor SQ22536 or a PKA inhibitor Rp-cAMP. However, pretreatment with another receptor antagonist CGRP8-37 did not suppress the increases in RSNA and MAP induced by IMD. Furthermore, IMD increased the cAMP level, which was inhibited by ADM22-52 pretreatment in the NTS. These results suggest that IMD participates in the sympathetic nerve activity and that central regulation of the cardiovascular system and a receptor-mediated cAMP-PKA signaling pathway is involved in such IMD-induced effects in the NTS.

Peripheral injection of IMD can relax blood vessels and decrease blood pressure by stimulating NO production [27]. Central treatment with IMD can activate the sympathetic nervous system and increase MAP [38]. In a rat renovascular hypertension model induced by 2-kidney 1-clip (2K1C) surgery, with bilateral paraventricular nucleus (PVN) microinjection of IMD, renal sympathetic nerve activity and MAP were decreased more in $2 \mathrm{~K} 1 \mathrm{C}$ than sham-operated 
rats and were prevented by pretreatment with the ADM receptor antagonist ADM22-52 or nonselective NOS inhibitor NG-nitro-L-arginine methyl ester [39]. Microinjection of IMD into the PVN increased the NO metabolite $\left(\mathrm{NO}_{x}\right)$ level in the PVN in $2 \mathrm{~K} 1 \mathrm{C}$ rats, which was prevented by ADM22-52 treatment. Chronic PVN infusion of IMD reduced but ADM22-52 increased blood pressure in conscious $2 \mathrm{~K} 1 \mathrm{C}$ rats. Therefore, IMD in the PVN inhibits sympathetic activity and attenuates hypertension in $2 \mathrm{~K} 1 \mathrm{C}$ rats, mediated by $\mathrm{ADM} 1$ and $\mathrm{ADM} 2$ receptors and its downstream NO. Therefore, the effect of IMD injection in the central nervous system on the sympathetic nervous system and blood pressure still needs further study.

As mentioned previously, the regulation of IMD on blood pressure is two-sided. Peripheral IMD can relax blood vessels and lower blood pressure, whereas central treatment with IMD usually activates the sympathetic nerve and elevates blood pressure. Downregulation of IMD as an endogenous regulatory peptide may have a role in the progression of renal impairment and hypertension [11]. IMD mRNA levels were significantly lower in SHRs than WKY rats in all renal portions. In the remnant kidneys of 5/6 nephrectomized rats, IMD mRNA levels were significantly decreased on days 3 and 56, whereas mRNA levels of CRLR, RAMP1, and RAMP2 were increased. Elevated IMD level and its receptor in the cardiovascular tissue may play an important role in the pathogenesis of spontaneous hypertension [17]. Immunoreactive IMD concentration was enhanced in the SHR myocardium, aortas and plasma, and IMD affected cAMP generation in the myocardium and aorta. In hypertensive DOCA-salt rats, IMD administration significantly lowered blood pressure, increased urine volume, and restored creatinine clearance [40]. IMD also greatly decreased superoxide formation and media thickness in the aorta. IMD gene delivery reduced vascular injury. Moreover, IMD gene transfer markedly decreased myofibroblast and collagen accumulation associated with decreased transforming growth factor- $\beta 1$ levels.

\subsection{IMD and vascular calcification}

Vascular calcification (VC) is highly associated with increased morbidity and mortality in patients with advanced chronic kidney disease [41]. Paracrine/autocrine factors such as vasoactive peptides are involved in VC development. Our laboratory [15] reported that IMD may be an endogenous vasoprotective factor for VC. Rat VC was induced by administration of vitamin D3 plus nicotine (VDN). VDN-treated rat aortas showed lower IMD content and increased expression of its receptors, along with increased vascular calcium deposition and alkaline phosphatase (ALP) activity. Low IMD levels were accompanied by increased calcium deposition in human atherosclerotic plaques. IMD greatly reduced vascular calcium deposition and ALP activity in VDN-treated rats. Concurrently, the loss of smoothmuscle lineage markers and matrix gamma-carboxyglutamic acid (Gla) protein (cMGP) in aortas was ameliorated by IMD in rats with VC, and the increased levels of phospho-Smad (1/5/8) and core binding factor $\alpha-1$ in calcified vasculature were also reduced. However, the inhibitory effects of IMD on VC were eliminated on pre-treatment with warfarin or small interfering RNA to reduce cMGP level. Therefore, reduced endogenous IMD levels are associated with increased mineralization in vivo, and administration of IMD inhibited VC development by increasing cMGP level. IMD1-53 attenuated vascular smooth muscle cell (VSMC) calcification by inhibiting endoplasmic reticulum stress (ERS) through cAMP/PKA signaling [28]. IMD1-53 treatment significantly alleviated the protein expression of ERS hallmarks activating transcription factor 4 (ATF4), ATF6, glucose-regulated protein 78 (GRP78) and GRP94. ERS occurred in early and late calcification of VSMCs but was inhibited by IMD1-53. These inhibitory effects of IMD1-53 were abolished by treatment with the PKA inhibitor H89. Pretreatment with IMD1-53 decreased the number of apoptotic VSMCs and downregulated the protein expression of cleaved caspase 12 and C/EBP homologous protein (CHOP) in calcified VSMCs. Concurrently, IMD1-53 restored the loss of VSMC lineage markers and ameliorated calcium deposition and ALP activity in calcified VSMCs.

\subsection{IMD and pulmonary arterial hypertension}

Recently, lots of data showed that IMD is involved in pulmonary arterial hypertension (PAH) pathogenesis. In $\mathrm{PAH}$ rats induced by hypoxia, mRNA levels and protein expressions of IMD, CRLR and RAMPs had changed apparently in right ventricle (RV), lung tissue and blood plasma [42-44]. In hypoxia rats, the concentration and mRNA level of IMD in plasma increased [42,44]. However, in lung tissue of hypoxia rats, IMD contents decreased but mRNA level of IMD was up-regulated; meanwhile, the mRNA levels of RAMP1 and CRLR were down-regulated [43,44]. In right ventricular tissue of hypoxia rats, IMD contents and mRNA levels of IMD and RAMP2 were higher [42]. Yang [45] discovered that in patients with chronic cor pulmonale at high altitude area, the levels of plasma IMD and mean pulmonary arterial pressure (mPAP) were markedly higher compared with those of healthy people.

Pang et al. [46] demonstrated that IMD alleviated the development of pulmonary hypertension and pulmonary vascular structural remodeling induced by high pulmonary blood flow through NOS/NO pathway. In high pulmonary blood flow rats by abdominal aorta and inferior vena cava shunting, mPAP, the ratio of right ventricular mass to left 
ventricular plus septal mass $\mathrm{RV} /(\mathrm{LV}+\mathrm{SP})$, the muscularization of small pulmonary vessels and relative medial thickness of pulmonary artery were all significantly increased. Meanwhile, the content of NO in the serum and pulmonary tissue homogenate and the expression of eNOS protein in rats of shunt group was all significantly decreased. IMD significantly decreased the mPAP and RV/(LV+SP), alleviated the changes of pulmonary vascular micro-structure, with the elevation of the content of NO in the serum and pulmonary tissue homogenate and the expression of pulmonary eNOS protein in shunting rats. Mao et al. [29] reported that IMD treatment alleviated PAH and prevented RV hypertrophy. IMD inhibited hypoxic pulmonary vascular remodeling as indicated by reduced wall thickness and increased lumen diameter of pulmonary arterioles, and decreased muscularization of distal pulmonary vasculature in hypoxia-exposed rats. IMD treatment inhibited pulmonary artery smooth muscle cell (PASMC) proliferation and promoted PASMC apoptosis. IMD treatment increased tissue level of constitutive NO synthase activity and tissue NO content in lungs, and enhanced L-arginine uptake into pulmonary vascular tissues. IMD treatment increased cellular levels of glucose-regulated protein (GRP) 78 and GRP94, two major markers of endoplasmic reticulum (ER) stress, and increased caspase-12 expression, the ER stress-specific caspase, in lungs and cultured PASMCs. These results demonstrated that IMD treatment attenuates hypoxic pulmonary vascular remodeling, and thereby hypoxic PAH mainly by inhibiting PASMC proliferation. Promotion of PASMC apoptosis may also contribute to the inhibitory effect of IMD. Activations of L-arginine-NO pathway and of ER stress-specific apoptosis pathway could be the mechanisms mediating the anti-proliferative and pro-apoptotic effects of IMD.

\section{Conclusion and future perspectives}

Growing evidence indicates that IMD is a vital regulatory factor of cardiovascular homoeostasis and is involved in the occurrence and development of various vascular diseases. IMD has a protective effect on atherosclerosis, vascular calcification, and hypertension and could be a novel target for treatment of cardiovascular system diseases. IMD exerts its functions through the common receptor of the CGRP superfamily of receptors, CRLR/RAMPs, and is nonselective to three kinds of CRLR/RAMPs. Therefore, investigators are interested in whether specific receptors of IMD exist in humans, which element in humans may influence the attraction of IMD and ADM to CRLR/RAMPs and how the change in attraction is regulated. IMD signal transduction pathways are complicated. Moreover, IMD1-47, IMD8-47, IMD1-53, and the different active segments of
IMD, have independent roles and also show synergistic or antagonistic interaction in regulating gene expression, biological effect, receptor binding, receptor signal transduction, genesis and development of disease (the theory of intramolecular regulation). The production of $I M D^{-/-}$animals, small interfering RNA, and specific inhibitor of IMD may play a vital role in research into the biological function of endogenous IMD and the molecular mechanisms involved. Confirmation of a crucial beneficial role of IMD should pave the way for translational research to the clinical arena. First, the potential of measuring IMD as a cardiovascular diseases biomarker could be addressed. Second, strategies could be devised to deliver IMD in the acute or chronic setting, such as infusion of the recombinant peptide or adenoviral vector-mediated delivery of the IMD gene to prevent or lessen the extent of vascular diseases.

This work was supported by the National Natural Science Foundation of China (91339203, 81270407, 81170082 to Qi YongFen).

1 Roh J, Chang CL, Bhalla A, Klein C, Hsu SY. Intermedin is a calcitonin/calcitonin gene-related peptide family peptide acting through the calcitonin receptor-like receptor/receptor activity-modifying protein receptor complexes. J Biol Chem, 2004, 279: 7264-7274

2 Kobayashi Y, Liu YJ, Gonda T, Takei Y. Coronary vasodilatory response to a novel peptide, adrenomedullin 2. Clin Exp Pharmacol Physiol, 2004, 31(Suppl 2): S49-S50

3 Taylor MM, Samson WK. Stress hormone secretion is altered by central administration of intermedin/adrenomedullin-2. Brain Res, 2005, 1045: 199-205

4 Taylor MM, Bagley SL, Samson WK. Intermedin/Adrenomedullin-2 inhibits growth hormone release from cultured, primary anterior pituitary cells. Endocrinology, 2006, 147: 859-864

5 Takahashi K, Kikuchi K, Maruyama Y, Urabe T, Nakajima K, Sasano H, Imai Y, Murakami O, Totsune K. Immunocytochemical localization of adrenomedullin 2/intermedin-like immunoreactivity in human hypothalamus, heart and kidney. Peptides, 2006, 27: 1383-1389

6 Hashimoto H, Hyodo S, Kawasaki M, Shibata M, Saito T, Suzuki H, Otsubo H, Yokoyama T, Fujihara H, Higuchi T, Takei Y, Ueta Y. Adrenomedullin 2 (AM2)/intermedin is a more potent activator of hypothalamic oxytocin-secreting neurons than AM possibly through an unidentified receptor in rats. Peptides, 2007, 28: 1104-1112

7 Song JQ, Teng X, Cai Y, Tang CS, Qi YF. Activation of Akt/GSK-3beta signaling pathway is involved in intermedin(1-53) protection against myocardial apoptosis induced by ischemia/reperfusion. Apoptosis, 2009, 14: 1061-1069

8 Pearson LJ, Yandle TG, Nicholls MG, Evans JJ. Intermedin (adrenomedullin-2): a potential protective role in human aortic endothelial cells. Cell Physiol Biochem, 2009, 23: 97-108

9 Du QX, Yue W, Wang YY. Effect and mechanism of intermedin in acute rat cardiac ischemic injury. Fa Yi Xue Za Zhi, 2011, 27: 164-168

10 Pires AL, Pinho M, Alves BS, Pinho S, Sena C, Seica RM, Leite-Moreira AF. Reverse myocardial effects of intermedin in pressure-overloaded hearts: role of endothelial nitric oxide synthase activity. J Physiol, 2013, 591(Pt 3): 677-687

11 Hirose T, Totsune K, Mori N, Mori T, Morimoto R, Metoki H, Asayama K, Kikuya M, Ohkubo T, Kohzuki M, Takahashi K, Imai Y. Expression of adrenomedullin 2/intermedin, a possible reno- 
protective peptide, is decreased in the kidneys of rats with hypertension or renal failure. Am J Physiol Renal Physiol, 2010, 299: F128-F134

12 Guo X, Schmitz JC, Kenney BC, Uchio EM, Kulkarni S, Cha CH. Intermedin is overexpressed in hepatocellular carcinoma and regulates cell proliferation and survival. Cancer Sci, 2012, 103: 1474-1480

13 Zhang W, Wang LJ, Xiao F, Wei Y, Ke W, Xin HB. Intermedin: a novel regulator for vascular remodeling and tumor vessel normalization by regulating vascular endothelial-cadherin and extracellular signal-regulated kinase. Arterioscler Thromb Vasc Biol, 2012, 32: 2721-2732

14 Du X, Cao Y, Xue P, Lin Z, Zeng Z, Xia Q. Protective effect of intermedin on myocardial cell in a rat model of severe acute pancreatitis. Cell Mol Biol Lett, 2011, 16: 462-476

15 Cai Y, Xu MJ, Teng X, Zhou YB, Chen L, Zhu Y, Wang X, Tang CS, Qi YF. Intermedin inhibits vascular calcification by increasing the level of matrix gamma-carboxyglutamic acid protein. Cardiovasc Res, 2010, 85: 864-873

16 Dai XY, Cai Y, Mao DD, Qi YF, Tang C, Xu Q, Zhu Y, Xu MJ, Wang X. Increased stability of phosphatase and tensin homolog by intermedin leading to scavenger receptor A inhibition of macrophages reduces atherosclerosis in apolipoprotein E-deficient mice. J Mol Cell Cardiol, 2012, 53: 509-520

17 Zeng Q, Yuan Y, Wang X, Wu HM, Fan L, Qi YF, Tang CS, Cai Y, Pan CS. Upregulated expression of intermedin and its receptor in the myocardium and aorta in spontaneously hypertensive rats. Peptides, 2009, 30: 391-399

18 Yang JH, Jia YX, Pan CS, Zhao J, Ouyang M, Yang J, Chang JK, Tang CS, Qi YF. Effects of intermedin(1-53) on cardiac function and ischemia/reperfusion injury in isolated rat hearts. Biochem Biophys Res Commun, 2005, 327: 713-719

19 Morimoto R, Satoh F, Murakami O, Totsune K, Suzuki T, Sasano H, Ito S, Takahashi K. Expression of adrenomedullin2/intermedin in human brain, heart, and kidney. Peptides, 2007, 28: 1095-1103

20 Conner AC, Hay DL, Howitt SG, Kilk K, Langel U, Wheatley M, Smith DM, Poyner DR. Interaction of calcitonin-gene-related peptide with its receptors. Biochem Soc Trans, 2002, 30: 451-455

21 Chen H, Wang X, Tong M, Wu D, Wu S, Chen J, Wang X, Wang X, Kang Y, Tang H, Tang C, Jiang W. Intermedin suppresses pressure overload cardiac hypertrophy through activation of autophagy. PLoS One, 2013, 8: e64757

22 Li P, Sun HJ, Han Y, Wang JJ, Zhang F, Tang CS, Zhou YB. Intermedin enhances sympathetic outflow via receptor-mediated cAMP/PKA signaling pathway in nucleus tractus solitarii of rats. Peptides, 2013, 47: 1-6

23 Aslam M, Pfeil U, Gunduz D, Rafiq A, Kummer W, Piper HM, Noll T. Intermedin (adrenomedullin2) stabilizes the endothelial barrier and antagonizes thrombin-induced barrier failure in endothelial cell monolayers. Br J Pharmacol, 2012, 165: 208-222

24 Yuan Y, Wang X, Zeng Q, Wu HM, Qi YF, Tang CS. Effects of continuous intermedin infusion on blood pressure and hemodynamic function in spontaneously hypertensive rats. J Geriatr Cardiol, 2012, 9: $17-27$

25 Pires AL, Pinho M, Sena CM, Seica R, Leite-Moreira AF. Intermedin elicits a negative inotropic effect in rat papillary muscles mediated by endothelial-derived nitric oxide. Am J Physiol Heart Circ Physiol, 2012, 302: H1131-H1137

26 Grossini E, Molinari C, Mary DA, Uberti F, Caimmi PP, Vacca G. Intracoronary intermedin 1-47 augments cardiac perfusion and function in anesthetized pigs: role of calcitonin receptors and beta-adrenoreceptor-mediated nitric oxide release. J Appl Physiol (1985), 2009, 107: 1037-1050

27 Kandilci HB, Gumusel B, Lippton H. Intermedin/adrenomedullin-2 (IMD/AM2) relaxes rat main pulmonary arterial rings via
cGMP-dependent pathway: role of nitric oxide and large conductance calcium-activated potassium channels (BK(Ca)). Peptides, 2008, 29: 1321-1328

28 Chang JR, Duan XH, Zhang BH, Teng X, Zhou YB, Liu Y, Yu YR, Zhu Y, Tang CS, Qi YF. Intermedin1-53 attenuates vascular smooth muscle cell calcification by inhibiting endoplasmic reticulum stress via cyclic adenosine monophosphate/protein kinase A pathway. Exp Biol Med (Maywood), 2013, 238: 1136-1146

29 Mao SZ, Fan XF, Xue F, Chen R, Chen XY, Yuan GS, Hu LG, Liu $\mathrm{SF}$, Gong YS. Intermedin modulates hypoxic pulmonary vascular remodeling by inhibiting pulmonary artery smooth muscle cell proliferation. Pulm Pharmacol Ther, 2014, 27: 1-9

30 Jolly L, March JE, Kemp PA, Bennett T, Gardiner SM. Mechanisms involved in the regional haemodynamic effects of intermedin (adrenomedullin 2) compared with adrenomedullin in conscious rats. $\mathrm{Br} \mathrm{J}$ Pharmacol, 2009, 157: 1502-1513

31 Smith RJ, Gao L, Bledsoe G, Chao L, Chao J. Intermedin is a new angiogenic growth factor. Am J Physiol Heart Circ Physiol, 2009, 297: H1040-H1047

32 Yang JH, Pan CS, Jia YX, Zhang J, Zhao J, Pang YZ, Yang J, Tang CS, Qi YF. Intermedin1-53 activates L-arginine/nitric oxide synthase/nitric oxide pathway in rat aortas. Biochem Biophys Res Commun, 2006, 341: 567-572

33 Abdelrahman AM, Pang CC. Vasodilator mechanism of intermedin/adrenomedullin-2 in anesthetized rats. Proc West Pharmacol Soc, 2007, 50: 43-46

34 Aslam M, Gunduz D, Schuler D, Li L, Sharifpanah F, Sedding D, Piper HM, Noll T. Intermedin induces loss of coronary microvascular endothelial barrier via derangement of actin cytoskeleton: role of RhoA and Rac1. Cardiovasc Res, 2011, 92: 276-286

35 Zhang X, Gu L, Chen X, Wang S, Deng X, Liu K, Lv Z, Yang R, He $\mathrm{S}$, Peng $\mathrm{Y}$, Huang D, Jiang W, Wu K. Intermedin ameliorates atherosclerosis in ApoE null mice by modifying lipid profiles. Peptides, 2012, 37: 189-193

36 Dai XY, Cai Y, Sun W, Ding Y, Wang W, Kong W, Tang C, Zhu Y, $\mathrm{Xu}$ MJ, Wang $\mathrm{X}$. Intermedin inhibits macrophage foam-cell formation via tristetraprolin-mediated decay of CD36 mRNA. Cardiovasc Res, 2014, 101: 297-305

37 Hirose T, Totsune K, Nakashige Y, Metoki H, Kikuya M, Ohkubo T, Hara A, Satoh M, Inoue R, Asayama K, Kondo T, Kamide K, Katsuya T, Ogihara T, Izumi S, Rakugi H, Takahashi K, Imai Y. Influence of adrenomedullin 2 /intermedin gene polymorphism on blood pressure, renal function and silent cerebrovascular lesions in Japanese: the Ohasama study. Hypertens Res, 2011, 34: 1327-1332

38 Taylor MM, Bagley SL, Samson WK. Intermedin/adrenomedullin-2 acts within central nervous system to elevate blood pressure and inhibit food and water intake. Am J Physiol Regul Integr Comp Physiol, 2005, 288: R919-R927

39 Zhou YB, Sun HJ, Chen D, Liu TY, Han Y, Wang JJ, Tang CS, Kang YM, Zhu GQ. Intermedin in paraventricular nucleus attenuates sympathetic activity and blood pressure via nitric oxide in hypertensive rats. Hypertension, 2014, 63: 330-337

40 Hagiwara M, Bledsoe G, Yang ZR, Smith RJ, Chao L, Chao J. Intermedin ameliorates vascular and renal injury by inhibition of oxidative stress. Am J Physiol Renal Physiol, 2008, 295: F1735-F1743

41 Schiffrin EL, Lipman ML, Mann JF. Chronic kidney disease: effects on the cardiovascular system. Circulation, 2007, 116: 85-97

42 Gong YS, Fan XF, Wu XM, Hu LG, Tang CS, Pang YZ, Qi YF. Changes of intermedin/adrenomedullin 2 and its receptors in the right ventricle of rats with chronic hypoxic pulmonary hypertension (in Chinese). Sheng Li Xue Bao, 2007, 59: 210-214

43 Fan XF, Huang P, Gong YS, Wu XM, Hu LG, Tian LX, Tang CS, Pang YZ. Changes of adrenomedullin 2/intermedin in the lung of rats 
with chronic hypoxic pulmonary hypertension (in Chinese). Zhongguo Ying Yong Sheng Li Xue Za Zhi, 2007, 23: 467-471

44 Wu DF, Tian LX, Fan XF, Wu XM, Hu LG, Zhang L, Huang P, Gong YS. Effect of hypoxia on the expressions of intermedin/adrenomedullin2 and its receptors in the lung of rats. J Chin Microcirc, 2007, 11: 94-97

45 Yang SY, Guo ZY, Feng ZE, Yan ZQ, Wang SL, Qi YS, He W, Tian
ZX, Yin H, Zhang Y. Changes of pulmonary vasoactive factors and its relation with pulmonary arterial hypertension in patients with chronic cor pulmonale at high altitude area. Chin J Lung Dis (Electronic Edition), 2011, 4: 211-215

46 Pang LL, Qi JG, Gao Y, Jin HF, Du JB. Intermedin alleviates pulmonary vascular structural remodeling induced by high pulmonary blood flow in rats. Basic Clin Med, 2013, 33: 1045-1049

Open Access This article is distributed under the terms of the Creative Commons Attribution License which permits any use, distribution, and reproduction in any medium, provided the original author(s) and source are credited. 